\title{
Die ,medizinische Notwendigkeit" humangenetischer Leistungen aus den Perspektiven des Faches und der Kostenträger
}

\author{
Jörg Schmidtke
}

Angenommen: 27. November 2020 / Online publiziert: 11. Dezember 2020

(C) Der/die Autor(en) 2020

Zusammenfassung In dieser Arbeit werden die Indikationskriterien zu humangenetischen Leistungen aus evidenzbasierter fachlicher Sicht dargestellt und die Pflicht zur Kostenübernahme oder die Berechtigung zu deren Ablehnung auf der Basis der gesetzlichen Grundlagen und der Rechtsprechung beleuchtet. Sie soll als eine Handlungsempfehlung sowohl für die indikationsstellende als auch für die gutachtlich tätige ärztliche Person dienen und als ein Appell an den Gesetzgeber, die Ungleichbehandlungen bezüglich humangenetischer Leistungen zu beseitigen.

\section{The "medical indication" for human genetics services from the providers' and payers' perspectives}

\begin{abstract}
In this work, the indication criteria for human genetic services are presented from an evidence-based professional point of view, and the obligation to assume costs or the authorization to reject them on the basis of legal principles and case law are examined. It is intended to serve as a recommendation for action both for the medical practitioner providing the indication and for the medical expert and as an appeal to the legislature to eliminate inequalities of treatment with regard to human genetic services.
\end{abstract}

\footnotetext{
J. Schmidtke $(\triangle)$

Institut für Humangenetik, Medizinische Hochschule Hannover,

Carl-Neuberg-Str. 1, 30625 Hannover, Deutschland

E-Mail: schmidtke.joerg@mh-hannover.de

amedes genetics, Georgstraße 50, 30159 Hannover, Deutschland
} 


\section{Einleitung}

Die ,medizinische Notwendigkeit“ einer ärztlichen oder ärztlich verordneten Leistung ist ein unbestimmter Rechtsbegriff, der im Einzelfall von den Kostenträgern überprüft werden darf und muss (Krimmel 1997). Denn nicht alles, was einer ärztlichen Person medizinisch sinnvoll oder geboten erscheint, ist aus der Perspektive der Kostenträger notwendig, und eine Indikationsstellung muss dann gegebenenfalls einer gutachtlichen Stellungnahme unterzogen werden (Nippert und Schmidtke 2012a, 2012b).

Begründungen für die medizinische Notwendigkeit humangenetischer Leistungen stehen in einem besonderen Spannungsverhältnis zwischen fachlicher Sicht und den rechtlichen Grundlagen, auf denen die Kostenträger ihre Entscheidungen begründen. Dies ist vor allem darauf zurückzuführen, dass das geltende Versicherungsvertragsgesetz (VVG) im Gegensatz zum Sozialgesetzbuch (SGB) fünftes Buch (V) einige der besonderen Merkmale humangenetischer Untersuchungen nicht in ausreichendem Maße berücksichtigt, insbesondere nicht ihre Rolle als Grundlage individueller Vorsorge (personalisierte Medizin auf der Grundlage persönlicher genetischer Daten) und ihre Relevanz für Dritte. Konflikte entstehen daher in erster Linie im Bereich der Privaten Krankenversicherung (PKV), weniger im Bereich der Gesetzlichen Krankenversicherung (GKV).

In dieser Arbeit werden die Indikationskriterien zu humangenetischen Leistungen aus evidenzbasierter fachlicher Sicht dargestellt und die Pflicht zur Kostenübernahme oder die Berechtigung zu deren Ablehnung auf der Basis der gesetzlichen Grundlagen und der Rechtsprechung beleuchtet. Sie soll als eine Handlungsempfehlung sowohl für die indikationsstellenden als auch für die gutachtlich tätige ärztliche Person dienen und als ein Appell an den Gesetzgeber, die eklatanten Ungleichbehandlungen bezüglich humangenetischer Leistungen zu beseitigen.

\section{Humangenetische Indikationskriterien aus fachlicher Sicht}

Indikationskriterien für humangenetische Untersuchungen wurden im deutschen Sprachraum von einer Kommission der Deutschen Gesellschaft für Humangenetik erstmals im Jahr 2007 ausformuliert (Propping et al. 2007; Schmidtke 2008). Sie wurden ab 2010 von der Europäischen Gesellschaft für Humangenetik übernommen und unter Bezeichnung „Clinical Utility Gene Cards“ im European Journal of Human Genetics (2020) fortlaufend publiziert. Die Indikationskriterien fußen ihrerseits auf dem ACCE ${ }^{1}$-Algorithmus des US-Center of Disease Control (2010) und repräsentieren damit einen breiten internationalen fachlichen Konsens. ${ }^{2}$

\footnotetext{
1 ACCE steht für ,,analytic validity, clinical validity, clinical utility and associated ethical, legal and social implications" von Gentests.

2 Fachliche Empfehlungen für genetische Untersuchungen werden krankheitsspezifisch in der OrphanetDatenbank für seltene Erkrankungen (Orphanet 2020) gelistet. Eine wichtige Ressource stellen die GeneReviews (2020) des US-NIH dar. Der zunehmenden Bedeutung von high-throughput Technologien bei der Mutationssuche im Zusammenhang mit genetisch heterogenen Erkrankungen (sog. ,next generation sequencing“, NGS) trägt insbesondere die ,panelapp“ des Genomics England Projekts (2020) Rechnung.
} 


\subsection{Settings}

Genetische Untersuchungen erfolgen danach in einem oder mehreren der vier Settings

- (Differential-)Diagnostik einer klinisch manifesten Erkrankung

- Prädiktive Diagnostik

- Risikoermittlung in Bezug auf Angehörige

- Vorgeburtliche Diagnostik

\subsection{Testqualitäten}

Jede Untersuchung wird in Bezug auf die Testqualitäten hin bewertet:

- Analytische Sensitivität (Anteil positiver Testergebnisse, wenn der gesuchte Genotyp vorhanden ist)

- Analytische Spezifität (Anteil negativer Testergebnisse, wenn der gesuchte Genotyp nicht vorhanden ist)

- Klinische Sensitivität (Anteil positiver Testergebnisse, wenn die Krankheit vorhanden ist)

- Klinische Spezifität (Anteil negativer Testergebnisse, wenn die Krankheit nicht vorhanden ist)

- Positiv klinisch prädiktiver Wert (Lebenszeitrisiko für das Auftreten der Krankheit, wenn der Test positiv ist)

- Negativ klinisch prädiktiver Wert (Wahrscheinlichkeit die Krankheit nicht zu entwickeln, wenn der Test negativ ist)

Innerhalb des aktuellen humangenetischen Testspektrums liegen analytische Sensitivität und Spezifität sowohl bei Einzelgenanalysen als auch bei genomischen Untersuchungsverfahren (Genpanels, Exomsequenzierungen) regelmäßig nahe $100 \%$. Klinische Sensitivität (,Mutations-Detektions-Wahrscheinlichkeit“) und Spezifität werden in hohem Maße und fast regelmäßig von der genetischen Heterogenität der Erkrankung beeinflusst und sind daher variabel. Unter dem Aspekt der ärztlichen Aufklärung sind die prädiktiven Werte von besonderem Interesse. Der positiv prädiktive Wert eines genetischen Tests wird oft von anderen nicht erfassbaren genetischen und nicht-genetischen Faktoren beeinflusst; wenn nicht alle Träger eines krankhaften Gens erkranken, spricht man von „reduzierter Penetranz“ des Genotyps - ein im medizinischen Alltag schwer vermittelbarer Aspekt. Der negativ prädiktive Wert wird in seiner Bedeutung im Zusammenhang mit einem familiär bedingten genetischen Risiko häufig unterschätzt; er liegt jedoch oft nahe $100 \%$ und entlastet damit die negativ getestete Person von der Sorge, selber erkranken zu können und damit von ansonsten erforderlichen, unter Umständen (auch den Kostenträger) belastenden und langfristigen Vorsorgemaßnahmen.

Diese Aussagen beziehen sich auf das Spektrum monogener, meist seltener Erkrankungen. Der individuelle prädiktive/prognostische Wert sogenannter ,polygener Risiko-Scores“ bei polygenen und multifaktoriellen Erkrankungen (den „Volks- 
krankheiten") ist so gering, dass für deren Ermittlung keine medizinische Notwendigkeit gesehen werden kann (Wald und Old 2019).

\subsection{Klinischer Nutzen}

Von zentraler Bedeutung für die Beurteilung der medizinischen Notwendigkeit einer genetischen Untersuchung ist der klinische (Zusatz-)Nutzen (,clinical utility“). Hier wird nach den eingangs beschriebenen ,Settings“ hin differenziert:

(Differential)diagnostisches Setting Die untersuchte Person ist klinisch betroffen.

- Kann eine Diagnosesicherung anders als durch genetische Untersuchungen erfolgen?

- Wie ist die Belastung alternativer Diagnosemethoden für den Patienten zu bewerten?

- Wie ist die Wirtschaftlichkeit alternativer Diagnosemethoden für den Kostenträger zu bewerten?

- Wird die Art der Behandlung des Krankheitsfalls durch die genetische Diagnostik beeinflusst (Prognose, Therapie, sonstiges Management)?

Prädiktives Setting Untersuchte Person ist frei von spezifischen Symptomen, trägt aber ein familiär bedingtes erhöhtes Risiko.

- Werden Lebensführung und Prävention durch das (positive oder negative) Ergebnis einer genetischen Diagnostik beeinflusst?

- Welche Optionen im Hinblick auf Lebensführung und Prävention stehen der Risikoperson offen, wenn keine genetische Diagnostik erfolgt?

\section{Setting Risikoermittlung in Bezug auf Angehörige}

- Klärt das Testergebnis beim Indexpatienten die genetische Situation in der Familie?

- Kann eine genetische Diagnostik beim Indexpatienten genetische oder andere Untersuchungen bei Familienangehörigen ersparen?

- Ermöglicht ein positives Testergebnis beim Indexpatienten eine prädiktive Diagnostik bei Angehörigen?

\section{Setting Pränataldiagnostik}

- Ermöglicht ein positives Testergebnis beim Indexpatienten eine vorgeburtliche Diagnostik?

Bei dieser Betrachtungsweise wird nicht die vorgeburtliche Diagnostik als solche sondern ihre Ermöglichung als „Nutzen“ betrachtet. Er besteht allein schon in der Option, ein genetisch bedingtes Risiko für ein erwartetes Kind präzisieren zu können. Ein Nutzen entsteht also nicht etwa erst dann, wenn unmittelbare medizinische Konsequenzen aus dem Ergebnis einer solchen Diagnostik gezogen werden sollen. Eine vorgeburtliche Diagnostik kann z. B. die werdenden Eltern frühzeitig auf die Geburt und die Pflege eines kranken Kindes hin vorbereiten. Schwangerschaftsbe- 
zogene Untersuchungen können diagnostisch oder prädiktiv sein, sie können auch Dritte einbeziehen, wie etwa den prospektiven Vater eines Kindes.

\section{Die medizinische Notwendigkeit von humangenetischen Untersuchungen aus der rechtlichen Perspektive der Kostenträger}

\subsection{Gesetzliche Krankenversicherung (GKV)}

In der GKV existiert der oft so apostrophierte „Leistungskatalog“ nicht als eine abgeschlossene Liste sondern als rahmenrechtliche Vorgabe im SGB V. Diese regelt, dass Leistungen ausreichend, zweckmäßig und wirtschaftlich sein müssen und das Maß des Notwendigen nicht überschreiten dürfen. Eine Entscheidung über das „,Maß des Notwendigen" muss die ärztliche Person in jedem Einzelfall treffen (Kleinken 2014) und damit grundsätzlich auch ggf. eine implizite Priorisierung bzw. Rationierung vornehmen (z. B. im Hinblick auf einen Schwellenwert eines genetischen Risikos, der eine genetische Untersuchung rechtfertigt) (Rogowski et al. 2014; Severin et al. $2015)^{3}$. Im Unterschied zu den für die PKV gültigen Regelungen des VVG (s. unten) tritt eine Leistungspflicht nicht erst bei dem Bestehen einer manifesten Erkrankung ein, sie betont vielmehr - gleich in $\S 1$ SGB V ,Solidarität und Eigenverantwortung“ - den Gedanken der (individuellen) Prävention: „Die Krankenversicherung als Solidargemeinschaft hat die Aufgabe, die Gesundheit der Versicherten zu erhalten, wiederherzustellen oder ihren Gesundheitszustand zu bessern. Das umfasst auch die Förderung der gesundheitlichen Eigenkompetenz und Eigenverantwortung der Versicherten. Die Versicherten sind für ihre Gesundheit mitverantwortlich; sie sollen durch eine gesundheitsbewusste Lebensführung, durch frühzeitige Beteiligung an gesundheitlichen Vorsorgemaßnahmen sowie durch aktive Mitwirkung an Krankenbehandlung und Rehabilitation dazu beitragen, den Eintritt von Krankheit und Behinderung zu vermeiden oder ihre Folgen zu überwinden. Die Krankenkassen haben den Versicherten dabei durch Aufklärung, Beratung und Leistungen zu helfen und auf gesunde Lebensverhältnisse hinzuwirken." (weitere Erläuterungen finden sich in $\S 11$ SGB V).

Im Zusammenhang mit humangenetischen Leistungen könnte der Appell an Solidarität, Eigenverantwortung und persönlichem Einsatz zur Prävention zunächst als Hinweis an die Versicherten (bzw. die sie behandelnden ärztlichen Personen) verstanden werden, angesichts wahrgenommener oder befürchteter genetischer Risiken eine

\footnotetext{
3 Restriktionen im Umfang humangenetischer Leistungen für GKV-Versicherte kommen z. B. darin zum Ausdruck, dass die Suche nach krankheitsverursachenden Genmutationen im EBM auf eine Gesamtlänge von 25.000 Basenpaaren begrenzt sind, deren Überschreitung einen umfänglichen und oft erfolglosen Antragsprozess bei der für den Patienten zuständigen Krankenkasse voraussetzt. Ansonsten werden humangenetische Leistungen (Beratung und Diagnostik) aktuell (aber zeitbegrenzt) extrabudgetär vergütet (GKV-Spitzenverband 2020).

Genetische Untersuchungen rechtfertigende Risikoschwellenwerte sind in der Fachliteratur teilweise leitliniengestützt. So wird ein sich aus der Familienvorgeschichte ergebendes a priori Risiko für erblichen Brustkrebs in Höhe von $10 \%$ oder mehr als Indikation für eine genetische Untersuchung angesehen (Deutsches Konsortium Familiärer Brust- und Eierstockkrebs 2020).
} 
Genetische Beratungseinrichtung aufzusuchen (bzw. an eine solche zu überweisen) ${ }^{4}$. Dies gilt insbesondere für die Settings „Prädiktive Diagnostik“ und „Risikoermittlung für Angehörige“. Die adäquate Nutzung genetischer Untersuchungsverfahren für die (Differential-)Diagnostik einer klinisch manifesten Erkrankung ist dagegen inzwischen fester Bestandteil verschiedener Fachdisziplinen geworden, die Indikationsstellung zur Pränataldiagnostik steht innerhalb frauenärztlicher Kompetenz, jeweils häufig in enger Kooperation mit Fachärzt*innen für Humangenetik.

\subsection{Private Krankenversicherung}

In der PKV ist die medizinische Notwendigkeit ärztlicher und damit auch humangenetischer Leistungen durch die Definitionen in den Musterbedingungen Krankheitskosten (Verband der Privaten Krankenversicherung 2020) beschrieben, deren gesetzliche Grundlage das Versicherungsvertragsgesetz (VVG, insbesondere $§ 192$ Abs. 1) bildet. „Versicherungsfall ist die medizinisch notwendige Heilbehandlung einer versicherten Person wegen Krankheit oder Unfallfolgen. Der Versicherungsfall beginnt mit der Heilbehandlung; er endet, wenn nach medizinischem Befund Behandlungsbedürftigkeit nicht mehr besteht. ... Als Versicherungsfall gelten auch ... Untersuchung und medizinisch notwendige Behandlung wegen Schwangerschaft und die Entbindung ... [sowie] ambulante Untersuchungen zur Früherkennung von Krankheiten nach gesetzlich eingeführten Programmen (gezielte Vorsorgeuntersuchungen) ...“ ( 1 Abs. 2 MB-KK). Die Formulierung ,wegen Krankheit“" lässt zwar offen, ob es sich hier um eine Krankheit handeln muss, die bei der versicherten Person klinisch (oder auch nur biochemisch oder bildgebend) manifest ist, oder ob es sich hier auch um eine Krankheit handeln könnte, für deren Eintreten ein spezifisch erhöhtes Risiko besteht (z. B. auf Grund der Familienanamnese und eines bekannten Erbgangs), welches durch einen prädiktiven Gentest präzisiert werden könnte. Allerdings geht die Rechtsprechung davon aus, dass nach dem Wortlaut des $§ 192$ Abs. 1 VVG Untersuchungen auf eine (noch) nicht manifeste Krankheit nur dann Gegenstand einer Heilbehandlung sein kann, wenn diese auf gesetzlich eingeführten Vorsorgeprogrammen beruht. Abgesehen vom Neugeborenen-Screening existieren jedoch keine gesetzlich eingeführten Vorsorgeprogramme bezüglich genetisch bedingter Erkrankungen, so dass prädiktive genetische Untersuchungen regelmäßig der privaten Vorsorge zugerechnet werden und daher nicht in die Leistungspflicht der PKV fallen (Landgericht Stuttgart 2012). Damit wäre die Voraussetzung für Leistungen der PKV im Zusammenhang mit genetischen Untersuchungen stets daran gebunden, dass die versicherte Person selbst manifeste Symptome einer Krankheit zeigt oder dass eine Schwangerschaft bereits eingetreten ist.

\footnotetext{
4 Im Gendiagnostikgesetz (GenDG) ist geregelt, dass eine Person, die genetisch untersucht werden soll (,,betroffene Person“), im Falle einer diagnostischen Untersuchung ärztlicherseits das Angebot einer genetischen Beratung erhalten soll ( $\$ 10$ Abs. 1) und im Fall einer prädiktiven oder pränatalen Untersuchung vorab genetisch beraten werden muss ( $\$ 10$, Abs. 2). Zu den prädiktiven Untersuchungen zählen nach der gesetzlichen Definition auch die Risikoermittlungen bei Angehörigen.
} 


\subsection{Beihilfe in Krankheits-, Pflege- und Geburtsfällen}

Beihilfe in Krankheits-, Pflege- und Geburtsfällen ist auf dem Wege einer Verordnung des Bundesministeriums des Inneren geregelt (Bundesministerium der Justiz und für Verbraucherschutz 2020). „Beihilfefähig sind grundsätzlich nur notwendige und wirtschaftlich angemessene Aufwendungen. ... Die Notwendigkeit von Aufwendungen für Untersuchungen und Behandlungen setzt grundsätzlich voraus, dass diese nach einer wissenschaftlich anerkannten Methode vorgenommen werden." (\$ 6 BBHV). Bezüglich Vorsorge und Früherkennung von Krankheiten finden im Beihilferecht die entsprechenden Regelungen des SGB V Anwendung (§ 41 BBHV). Das Bundesverwaltungsgericht (2017) hat in diesem Zusammenhang geurteilt, dass eine Krankheit im beihilferechtlichen Sinn auch dann vorliegen kann ,wenn die auf Tatsachen gestützte konkrete Gefahr einer schwerwiegenden Gesundheitsschädigung besteht", aber die Störung selbst noch nicht ausgebrochen ist. In diesem Fall ging es um die Beihilfefähigkeit einer prophylaktischen Mastektomie bei einer bereits erwiesenen Trägerin einer Mutation in einem Brustkrebs-assoziierten Gen (und nicht um die Frage der medizinischen Notwendigkeit der vorausgehenden genetischen Untersuchung).

\section{Genetische Untersuchungen im diagnostischen Setting}

Unter der Voraussetzung, dass ein wissenschaftlich fundiertes Untersuchungsverfahren zur Verfügung steht (s. oben „Testqualitäten“), stehen GKV, PKV und Beihilfestellen im diagnostischen Setting grundsätzlich in der Leistungspflicht, die nur durch ein Wirtschaftlichkeitsgebot (GKV) bzw. ein Übermaßverbot (PKV) eingeschränkt wird. Wenn bei einer manifesten Erkrankung eine genetische Untersuchung erfolgen soll, sollte sich der Arzt an den oben genannten Kriterien für einen klinischen (Zusatz-)Nutzen einer solchen Maßnahme orientieren.

Die Sicherung einer Diagnose erfolgt in der Medizin nahezu regelmäßig in der Zusammenschau subjektiver und objektiver klinischer Symptome, den Ergebnissen von bildgebenden, elektrophysiologischen oder histopathologischen Untersuchungen sowie klinisch-chemischen oder immunologischen Laborwerten. Welche dieser Bausteine im Einzelfall notwendiger Bestandteil der Diagnosefindung sind, muss stets abgewogen und ein Übermaß bzw. Unwirtschaftlichkeit vermieden werden. Maßstab für das notwendige Ausmaß einer differentialdiagnostischen Abklärung ist dessen Relevanz für Prognose und Therapie. Die Indikationsstellung zu einer genetischen Untersuchung sollte in diesen Abwägungsprozess eingefügt werden. Die ärztliche Person muss sich fragen, ob genetische Untersuchungen zu bereits vorliegenden Daten einen hinreichenden Zusatznutzen entfalten, oder ob sie sogar eher am Anfang des diagnostischen Prozedere stehen sollten und damit andere Untersuchungen ersparen. Letzteres ist gerade im Bereich der Seltenen Erkrankungen häufig der Fall, also dem Patientengut, mit dem Humangenetiker*innen weit überwiegend befasst ist und welches häufig durch kostentreibende vorausgehende „Patient*innenodysseen“ charakterisiert ist (Global Commission 2020). Die Kosteneffektivität genetischer Untersuchungen als primär-diagnostisches Instrument konnte 
unter anderem am Beispiel der dilatativen Kardiomyopathie gezeigt werden (Catchpool et al. 2019).

Neben der Frage der Wirtschaftlichkeit spielt auch die Frage der Belastung der Patient*innen durch verschiedene Untersuchungsverfahren eine tragende Rolle. Genetische Untersuchungen erfolgen regelmäßig an einer Blutprobe oder einem Mundschleimhautabstrich, die verglichen z. B. mit Biopsien oder Belastungstests, nur minimal körperlich belastend sind.

Eine Sonderstellung nehmen pharmakogenetische Untersuchungen ein, die nach $\S 3$ Nr. 7 lit. c GenDG, ebenfalls zu den diagnostischen genetischen Untersuchungen zählen. Die Pharmakogenetik befasst sich mit dem Einfluss der genetischen Ausstattung von Patienten auf die Wirkung von Arzneimitteln. In Deutschland werden die medizinisch-wissenschaftlichen Rahmenbedingungen für eine pharmakogenetische Diagnostik in einer Richtlinie der Gendiagnostikkommission am Robert-KochInstitut festgelegt (Robert-Koch Institut 2017). Diese Richtlinie nimmt selber keine Nutzenbewertung zum Zweck des Nachweises pharmakogenetischer Eigenschaften (und damit einer etwaigen Begründung einer medizinischen Notwendigkeit) vor. Sie verweist für diesen Zweck vielmehr auf einschlägige Datenbanken, die den aktuellen Stand der medizinischen Wissenschaft und Technik widerspiegeln. An erster Stelle ist hier das Clinical Pharmacogenetics Implementation Consortium (CPIC) des Pharmacogenetics Research Network (2020) zu nennen. Es listet laufend aktualisiert Gen-Arzneistoff-Kombinationen auf, die für eine pharmakogenetische Untersuchung im Einzelfall in Frage kommen könnten und gibt eine Bewertung des klinischen Nutzens auf der Basis der verfügbaren Evidenz ab. „Die CPIC Empfehlungen stellen den gegenwärtig solidesten Ausgangspunkt für auf Pharmakogenetik beruhende Auswahl und Dosierung ausgewählter Arzneistoffe dar“ (Cascorbi 2017). Aktuell listet das CPIC 416 pharmakogenetisch relevante Gen-Medikament-Paare mit unterschiedlichen Evidenzleveln für die praktisch-medizinische Relevanz. Ein Beispiel für eine zwingende medizinische Indikation für eine pharmakogenetische Untersuchung ist die Genotypisierung des $H L A-B$-Gens bei der HIV-Therapie mit Abacavir. Etwa $6 \%$ aller Patienten tragen eine $H L A-B$-Variante, die mit einer u. U. lebensbedrohlichen Abacavir-Hypersensitivität einhergeht.

Ein weiterer Sonderfall entsteht, wenn die Untersuchung Dritter erforderlich wird, um ein Untersuchungsergebnis bei einer erkrankten Person korrekt zu interpretieren. So ist der Einschluss der Eltern oft Bestandteil einer genetischen Untersuchung eines erkrankten Kindes.

\section{Prädiktives Setting}

Es wurde oben bereits herausgearbeitet, dass im Zusammenhang mit prädiktiven genetischen Untersuchungen rechtliche Unterschiede zwischen den Leistungspflichten der GKV und der PKV bestehen. Während die individuelle Nutzung prädiktiver Gentests von der GKV geradezu gefordert zu werden scheint, ist sie auf der Basis richterlicher Interpretation der aktuellen Gesetzeslage in der PKV unzulässig.

In der Praxis handeln die privaten Versicherer jedoch uneinheitlich. Ein Beispiel dafür sind prädiktive genetische Untersuchungen beim familiären Brust- und 
Eierstockkrebs. Bereits im Jahre 2006 hatte der Verband der Privaten Krankenversicherung e. V. (PKV-Verband) eine Vereinbarung mit einem universitären Zentrum gegründet, nach der prädiktive genetische Untersuchungen bei dieser Fragestellung grundsätzlich übernommen wurden. Die Vereinbarung ist so ausgestaltet, dass ihr alle Mitgliedsunternehmen des PKV-Verbandes beitreten können (aber nicht müssen). Nur Patienten, deren Versicherer beigetreten ist, können die unten beschriebenen Leistungen im Rahmen der Vereinbarung in Anspruch nehmen. Auf Seiten der Leistungserbringer sind derzeit 17 universitäre Zentren dem Vertrag beigetreten. Hierbei handelt es sich um Mitglieder des Deutschen Konsortiums Familiärer Brust- und Eierstockkrebs. Aus rechtlicher Sicht birgt diese Vereinbarung einige Ungereimtheiten. Zum einen ist nicht hinreichend transparent, welche Versicherer der Vereinbarung beigetreten sind (und damit die Regelungen des § 1 Abs. 2 MBKK faktisch umgehen). Zum anderen würde eine Begrenzung der Kostenübernahme auf einige wenige Leistungsanbieter der freien Arztwahl nach § 4 Abs. 2 MB-KK widersprechen. Des weiteren vermag es nicht einzuleuchten, dass prädiktive genetische Untersuchungen auf Patient*innen mit dieser einen Erkrankung beschränkt werden sollen und Patient*innen mit anderen Erkrankungen vorenthalten werden, darunter z.B. erblichen Herzerkrankungen, bei denen der klinische Nutzen einer solchen Untersuchung in gleicher Weise erwiesen und in einschlägigen Leitlinien dargelegt ist (Schulze-Bahr et al. 2015). Das American College of Medical Genetics (NCBI 2020) hat eine Liste von derzeit 59 Gen-Krankheitsassoziationen erstellt, die hinsichtlich vorsorglicher Behandlungsmöglichkeiten von Trägern pathogener Mutationen besonders gut evaluiert sind. Es handelt sich hierbei überwiegend um erbliche Krebserkrankungen (neben Brustkrebs z.B. Darm- und Schilddrüsenkrebs) und Herz-Kreislauferkrankungen (z. B. die thorakale Aortensyndrome), daneben u. a. die familiäre Hypercholesterinämie und die maligne Hyperthermie. In der Tat erkennen private Versicherungsunternehmen das Problem einer unzulässigen Ungleichbehandlung ihrer Versicherten, unterziehen Fälle dieser Art einer Einzelfallprüfung und entscheiden häufig positiv, obgleich die MB-KK und das VVG keine Grundlage für solche Entscheidungen geben. Es ist allerdings unbekannt, in welchem Umfang Versicherungsnehmer bzw. die sie behandelnden ärztlichen Personen den manchmal mühevollen Weg der Erwirkung einer Kostenerstattung überhaupt beschreiten.

Würde eine manifest bestehende Krankheit regelmäßige Voraussetzung für eine humangenetische Leistung sein, so wäre nicht einmal eine genetische Beratung erstattungspflichtig, die sich eine gesunde Person wegen eines familiär bedingt erhöhten genetischen Risikos einholt. Dem Autor dieser Arbeit ist jedoch kein Fall bekannt, in dem ein solcher Erstattungsanspruch verweigert worden wäre. Auch in diesem Punkt folgen Versicherer also nicht dem Wortlaut des MB-KK bzw. seiner richterlichen Interpretation (s. oben).

Die hier aufgezeigten Widersprüche könnten der Gesetzgeber und der PKV-Verband durch entsprechende Anpassungen des VVG leicht ändern. Einzig und allein müsste der Möglichkeit der individuellen Vorsorgeuntersuchung und Früherkennung bei erhöhtem genetisch bedingtem Risiko Raum gegeben werden.

Im Zuge einer solchen Gesetzesreform sollte auch ein weiteres, immer wieder aufkommendes Problem über die Zuständigkeit der Kostenträger bei prädiktiven genetischen Untersuchungen gelöst werden: Ein prädiktiver Gentest bei einem ge- 
sunden Angehörigen einer erkrankten Person setzt in der Regel die Bekanntheit der ursächlichen Genmutation bei der erkrankten Person voraus. Die unter Umständen aufwändige Suche nach dieser Mutation sollte von dem Kostenträger der Person übernommen werden, in deren Interesse die Identifikation der Mutation steht.

\section{Setting: Risikoermittlung in Bezug auf Angehörige}

Dieses Setting erscheint deswegen als ein separates Szenarium, weil gelegentlich genetische Untersuchungen bei einer erkrankten Person ausschließlich im Interesse Dritter erfolgen, um diesen einen Gentest zu ermöglichen oder für sie gar nicht erst erforderlich machen. Selbst dann, wenn aus einer genetischen Untersuchung keinerlei Konsequenzen für die Prognose oder die Therapierbarkeit für die erkrankte Person selbst entstehen, kann sie für einen Angehörigen wertvolle Informationen liefern, z. B. für dessen Familienplanung oder individuelle Vorsorge. In dieses Setting gehören auch Untersuchungen zum Erbgang einer Erkrankung in einer Familie, die unter Umständen den Angehörigen (und den Kostenträgern) genetische Untersuchungen vollkommen ersparen. Sowohl für eine GKV- als auch für eine PKVversicherte erkrankte Person ergäbe sich auf der Basis der aktuellen Gesetzeslage jedoch ein Behandlungsübermaß. Diese Situation kann - wie im vorausgehenden Abschnitt angeregt - zumindest teilweise dadurch gelöst werden, dass der Kostenträger der Person eintritt, in deren Interesse die genetische Untersuchung (oder ihre Vermeidung) steht.

\section{Pränatales Setting}

Sowohl in der GKV als auch in der PKV gehören schwangerschaftsbezogene Maßnahmen grundsätzlich zu den Leistungspflichten. In analoger Weise zu der Diskussion in Abschn. 3.2 ist davon auszugehen, dass in der PKV, nicht jedoch in der GKV eine Leistungspflicht für genetische Untersuchungen ,wegen Schwangerschaft“ das Bestehen einer solchen voraussetzt. Wenn dies der Fall ist, sind Kostenerstattungen regelmäßig unproblematisch. Die Konsequenzen der Notwendigkeit der bestehenden Schwangerschaft für schwangerschaftsbezogene Gentests wurden bereits früher eingehend thematisiert (Grobenski et al. 2010). Bei einem Bestehen genetischer Risiken (z.B. in der eigenen oder familiären Vorgeschichte), die eine vorgeburtliche Diagnostik und ggf. einen Schwangerschaftsabbruch aus mütterlicher Perspektive rechtfertigen würden, müsste danach eine Frau mit Kinderwunsch erst tatsächlich schwanger werden (sozusagen ,,auf Probe“), um überhaupt einen Erstattungsanspruch auf die Kosten zu erwirken, die eine Abklärung solcher Risiken durch eine genetische Untersuchung verursachen. Dies ist eine erkennbar abwegige Situation, die möglicherweise im Wege von Einzelfallentscheidungen befriedigend gelöst werden kann, zeigt aber doch auf, dass die Gesetzeslage auch in diesem Punkt geändert werden muss. 


\section{Fazit}

Trotz des hohen Stellenwerts, den diagnostische und prädiktive humangenetische Untersuchungen inzwischen im medizinischen Alltag vieler Fachgebiete einnehmen, werden Kostenerstattungen auf Grund divergenter bzw. nicht zureichender gesetzlicher Voraussetzungen in unbefriedigender Weise gehandhabt. Ein Weg zu einer Lösung dieser Situation könnte darin bestehen, dass die in der wissenschaftlichen Literatur gut dokumentierten Algorithmen allgemeine Akzeptanz erfahren und der Gesetzgeber die bestehenden Ungleichheiten in der Möglichkeit zur Nutzung einer auf genetischen Daten beruhenden personalisierten Medizin behebt.

Danksagung Ich danke Dr. Ralf Glaubitz und Prof. Dr. Gerhard Wolff für die Durchsicht des Manuskripts.

Funding Open Access funding enabled and organized by Projekt DEAL.

Open Access Dieser Artikel wird unter der Creative Commons Namensnennung 4.0 International Lizenz veröffentlicht, welche die Nutzung, Vervielfältigung, Bearbeitung, Verbreitung und Wiedergabe in jeglichem Medium und Format erlaubt, sofern Sie den/die ursprünglichen Autor(en) und die Quelle ordnungsgemäß nennen, einen Link zur Creative Commons Lizenz beifügen und angeben, ob Änderungen vorgenommen wurden.

Die in diesem Artikel enthaltenen Bilder und sonstiges Drittmaterial unterliegen ebenfalls der genannten Creative Commons Lizenz, sofern sich aus der Abbildungslegende nichts anderes ergibt. Sofern das betreffende Material nicht unter der genannten Creative Commons Lizenz steht und die betreffende Handlung nicht nach gesetzlichen Vorschriften erlaubt ist, ist für die oben aufgeführten Weiterverwendungen des Materials die Einwilligung des jeweiligen Rechteinhabers einzuholen.

Weitere Details zur Lizenz entnehmen Sie bitte der Lizenzinformation auf http://creativecommons.org/ licenses/by/4.0/deed.de.

\section{Literatur}

Bundesministerium der Justiz und für Verbraucherschutz (2020). https://www.gesetze-im-internet.de/ bbhv/, Zugegriffen: 6. Nov. 2020

Bundesverwaltungsgericht: Urteil vom 28.09.2017; BVerwG 5C 10.16 (2017)

Cascorbi, I.: Pharmakogenetik. Aktueller Stand - Fakten und Fiktionen. medgen 29, 389-396 (2017)

Catchpool, M., Ramchand, J., Martyn, M., Hare, D.L., James, P.A., Trainer, A.H., Knight, J., Goranitis, I.: A cost-effectiveness model of genetic testing and periodical clinical screening for the evaluation of families with dilated cardiomyopathy. Genet. Med. 21, 2815-2822 (2019)

Centers for Disease Control and Prevention: ACCE model list of 44 targeted questions (2010). https:// www.cdc.gov/genomics/gtesting/acce/acce_proj.htm, Zugegriffen: 6. Nov. 2020

Clinical Pharmacogenetics Implementation Consortium: Clinical Pharmacogenetics Implementation Consortium (2020). https://cpicpgx.org/genes-drugs/, Zugegriffen: 6. Nov. 2020

Deutsches Konsortium Familiärer Brust- und Eierstockkrebs: Deutsches Konsortium Familiärer Brustund Eierstockkrebs (2020). https://www.konsortium-familiaerer-brustkrebs.de/betreuungskonzept/ molekulare-diagnostik/indikationen-gentest/, Zugegriffen: 6. Nov. 2020

European journal of human genetics: clinical utility gene cards (2020). https://www.nature.com/collections/ lmbjcgchnm, Zugegriffen: 6. Nov. 2020

GeneReviews (2020). https://www.ncbi.nlm.nih.gov/books/NBK1116/, Zugegriffen: 6. Nov. 2020

Genomics England (2020). https://panelapp.genomicsengland.co.uk/, Zugegriffen: 6. Nov. 2020

GKV-Spitzenverband (2020). https://www.gkv-spitzenverband.de/presse/themen/verguetung_aerztlicher_ leistungen/s_thema_aerzteverguetung.jsp, Zugegriffen: 6. Nov. 2020

Global Commission: Global Commission (2020). https://globalrarediseasecommission.com/Report/, Zugegriffen: 6. Nov. 2020 
Grobenski, Z., Graf von der Schulenburg, J.-M., Schmidtke, J.: Schwangerschaftsbezogene prädiktive Gentests als Leistung der privaten Krankenversicherung. MedR 28, 387-396 (2010)

Kleinken, B.: Wer bestimmt, was „,medizinisch notwendig“ ist? AAA Abrech. Aktuell 8, 14 (2014)

Krimmel, L.: Ambulante Versorgung unter Budgetzwang: was ist „,medizinisch notwendig“? Dtsch. Arztebl. 94, A-20 (1997)

Landgericht Stuttgart: Urteil vom 19.12.2012; 13 S 131/12 (2012)

NCBI (2020). https://www.ncbi.nlm.nih.gov/clinvar/docs/acmg/, Zugegriffen: 6. Nov. 2020

Nippert, R.P., Schmidtke, J.: Versorgungsqualität bei genetisch bedingten Erkrankungen - Sekundäranalyse humangenetischer Gutachten. Z Evid Fortbild Qual Gesundhwes 106, 217-223 (2012a)

Nippert, R.P., Schmidtke, J.: Was wollen Versicherungen wissen? Auskunftsersuchen privater Krankenversicherungen zu humangenetischen Leistungen. Versicherungsmedizin 64, 12-16 (2012b)

Orphanet (2020). www.orpha.net, Zugegriffen: 6. Nov. 2020

Propping, P., Schmidtke, J., Schwinger, E., Wieacker, P.: Zur Definition des klinischen Nutzens genetischer Diagnostik bei erblichen Krankheiten - Eckpunkte für eine Kriterienentwicklung. medgen 2, 274-275 (2007)

Robert-Koch Institut (2017). https://www.rki.de/DE/Content/Kommissionen/GendiagnostikKommission/ Richtlinien/RL-WirkungArzneimittel.pdf?_blob=publicationFile, Zugegriffen: 10. Okt. 2020

Rogowski, W.H., Grosse, S.D., Schmidtke, J., Marckmann, G.: Criteria for fairly allocating scarce healthcare resources to genetic tests: which matter most? Eur. J. Hum. Genet. 22, 25-31 (2014)

Schmidtke, J.: Gentests: Auf dem Prüfstand der Genetiker. Dtsch Ärztebl. 105, 1830-1834 (2008)

Schulze-Bahr, E., Klaassen, S., Abdul-Khaliq, H., Schunkert, H.: Gendiagnostik bei kardiovaskulären Erkrankungen - Positionspapier der Deutschen Gesellschaft für Kardiologie (DGK) und der Deutschen Gesellschaft für Pädiatrische Kardiologie (DGPK). Kardiologe 9, 213-243 (2015)

Severin, F., Borry, P., Cornel, M.C., Daniels, N., Fellmann, F., Hodgson, V.S., Howard, H.C., John, J., Kääriäinen, H., Kayserili, H., Kent, A., Koerber, F., Kristoffersson, U., Kroese, M., Lewis, C., Marckmann, G., Meyer, P., Pfeufer, A., Schmidtke, J., Skirton, H., Tranebjærg, L., Rogowski, W.H., EuroGentest and ESHG/PPPC Priority Consortium: Points to consider for prioritizing clinical genetic testing services: a European consensus process oriented at accountability for reasonableness. Eur. J. Hum. Genet. 23, 729-735 (2015)

Verband der Privaten Krankenversicherung (2020). https://www.pkv.de/service/rechtsquellen/muster bedingungen/mb_kk_2009.pdf, Zugegriffen: 6. Nov. 2020

Wald, N.J., Old, R.: The illusion of polygenic risk prediction. Genet. Med. 21, 1705-1707 (2019) 